\title{
Comprehensive analysis of gene expression profiles associated with proliferative diabetic retinopathy
}

\author{
MENG-TING GONG ${ }^{1 *}$, WEN-XING LI ${ }^{2 *}$, QING ZHANG ${ }^{3 *}$, WEN-WEN LV ${ }^{4,5}$, ZHENG-HONG HE $^{6}$, \\ SHU-LI ZHOU ${ }^{6}$, HUI ZHANG ${ }^{6}$, JING WANG ${ }^{6}$ and KAN HE $^{1,6}$ \\ ${ }^{1}$ Center for Stem Cell and Translational Medicine, School of Life Sciences, Anhui University, \\ Hefei, Anhui 230601; ${ }^{2}$ State Key Laboratory of Genetic Resources and Evolution, \\ Kunming Institute of Zoology, Chinese Academy of Sciences, Kunming, Yunnan 650223; \\ ${ }^{3}$ Department of Ophthalmology, The Second Hospital of Anhui Medical University, Hefei, Anhui 230601; \\ ${ }^{4}$ Hongqiao International Institute of Medicine, Shanghai Tongren Hospital/Faculty of Public Health; \\ ${ }^{5}$ Clinical Research Center, Shanghai Jiao Tong University School of Medicine, Shanghai 200025; \\ ${ }^{6}$ Department of Biostatistics, School of Life Sciences, Anhui University, Hefei, Anhui 230601, P.R. China
}

Received March 19, 2018; Accepted July 26, 2018

DOI: $10.3892 /$ etm.2018.6635

\begin{abstract}
Proliferative diabetic retinopathy (PDR) is characterized by neovascularization on the surface of the retina or the optic disc, which is associated with environmental and genetic factors. However, its regulatory mechanism remains to be fully elucidated, particularly at a multiomics level. In the present study, a comprehensive analysis was performed of the gene expression profile of fibrovascular membranes (FVMs) associated with PDR, including an analysis of differentially expressed genes, functional enrichment, and regulation of transcription factors (TFs). As a result, novel marker genes of PDR were identified, including flavin containing monooxygenase 2. Furthermore, several common or specific genes, pathways and TFs have been recovered for active and inactive FVMs. In the present study, lymphoid enhancer binding factor 1 (LEF1) was identified as an upregulator in active and inactive FVMs, which is capable of activating or repressing target genes, including claudin 2, secreted phosphoprotein 1 (SPP1), and aristaless-like homeobox 4. It was demonstrated that the Wnt/ $\beta$-catenin effector LEF1 regulating SPP1 is potentially important in PDR. The results of the present study may provide novel insights into the molecular mechanisms underlying the pathophysiology of PDR.
\end{abstract}

Correspondence to: Dr Kan He, Center for Stem Cell and Translational Medicine, School of Life Sciences, Anhui University, 111 Jiulong Road, Hefei, Anhui 230601, P.R. China

E-mail: hekan_803@163.com

*Contributed equally

Key words: proliferative diabetic retinopathy, genes, pathways, transcription factors, LEF1

\section{Introduction}

Diabetic retinopathy (DR) is a common blinding complication in patients with type 1 and type 2 diabetes mellitus $(1,2)$. Proliferative DR (PDR) is a more serious stage of DR, which is characterized by neovascularization on the surface of the retina or the optic disc (3). The newly-formed vessels are prone to hemorrhaging, which threatens the vision of the sufferer and eventually culminates in blindness. PDR is associated with environmental and genetic factors. The length of time patients have suffered with diabetes, glycemic control, hypertension, and other environmental factors are well-established risk factors of PDR (4). Genetic variations may explain some of the heterogeneity in the development of PDR. Several candidate genes have been implicated in the pathogenesis of PDR in patients with type 2 diabetes (5). For example, vascular endothelial growth factor (VEGF) was found to be overexpressed in fibrovascular membranes (FVMs) in patients with PDR, suggesting that it may contribute to the development of PDR (6). Gene polymorphisms of other growth factors, including basic fibroblast growth factor and insulin-like growth factor have also been shown to be important in the pathogenesis of $\operatorname{PDR}(7,8)$. The association between single-nucleotide polymorphisms of oxidative stress genes and PDR in type 2 diabetes has been reported in a number of previous studies, including manganese superoxide dismutase, catalase myeloperoxidase, glutathione S-transferase, NADPH oxidase, endothelial nitric oxide synthase and inducible nitric oxide synthase (9-11).

However, the exact pathogenesis of PDR remains to be elucidated. At present, an increasing number of gene expression profiling studies have been performed to reveal the genetic mechanisms of PDR $(3,12,13)$. The transcriptomics datasets using the platforms of microarrays or RNA-sequencing are available in public databases, including the functional genomics data repository of Gene Expression Omnibus (GEO) and Sequence Read Archive (14). In the present study, 
microarray datasets of gene expression in FVMs excised from patients with proliferative diabetic retinopathy were utilized to comprehensively analyze the molecular expression patterns of PDR.

\section{Materials and methods}

Microarray data collection and preprocessing. In order to investigate the regulatory mechanisms of PDR at the transcriptomic level, gene expression profiling datasets were retrieved from the GEO. Finally, the raw data of GSE60436 contributed by Ishikawa et al, which is the gene expression profile of FVMs associated with PDR, was selected and downloaded (3). In this array, there were a total of nine RNA samples. Of these, three control RNA samples were from the human retina obtained from Clontech Laboratories, Inc. (Mountainview, CA, USA), and six RNA samples were obtained from the retinal surface with horizontal scissors of patients with PDR undergoing pars plana vitrectomy, and were classified into active and inactive samples according to the clinical findings of neovascularization (NV) in the FVMs, with three samples per group. The active FVMs represent samples with the presence of NV in the membranes, and the inactive FVMs represent samples with an absence of NV in the membranes. The Illumina HumanWG-6 v3.0 Expression BeadChip (GPL6884; Illumina, Inc., San Diego, CA, USA) platform was used to screen PDR-associated genes. The data preprocessing was performed by $\mathrm{R} v 3.2 .2$ (https://www.r-project.org/). The Robust Multichip Average algorithm in the oligo package was used to normalize the raw expression data and generate normalized gene expression intensity (15).

Differentially expressed gene (DEG) analysis. The analysis of DEGs was performed using R v3.2.2 and Bioconductor libraries (http://www.bioconductor.org/). The empirical Bayes algorithm (function 'eBayes') within the Limma package was used to detect DEGs between the case samples, including the active or inactive samples and the controls. Upregulated genes were considered as a logarithmic transformed fold-change $\log 2(\mathrm{FC}) \geq 1$ and a false discovery rate (FDR) adjusted P-value $\leq 0.05$. Downregulated genes were considered as $\log 2(\mathrm{FC}) \leq-1$ and an FDR P-value $\leq 0.05$. Differential expression analysis in the two comparisons for the active and inactive FVMs were performed.

Kyoto encyclopedia of genes and genomes (KEGG) pathway enrichment analysis. The Database for Annotation, Visualization, and Integrated Discovery Bioinformatics Resource 6.7 (https://david.ncifcrf.gov/) was used to perform KEGG pathway enrichment analysis (16). The inputs were utilized from the list of DEGs. An enriched pathway where $\mathrm{P}<0.05$ was considered to indicate a statistically significant difference. InteractiVenn (http://www.interactivenn.net/) was utilized to draw a Venn diagram in order to illustrate the overlapping genes or pathways between active and inactive regulation.

Targeted genes by transcription factor (TF) analysis. The union set of the differentially expressed target genes in total and the subgroups was obtained and IneractiVenn software was used to illustrate the results. TF analysis was performed by integrating the database of TRANSFAC ${ }^{\circledR} 7.0$ (http://genexplain.com/transfac/), which provides data on eukaryotic TFs, their experimentally-proven binding sites, consensus binding sequences (positional weight matrices), and regulated genes $(17,18)$.

\section{Results and Discussion}

DEGs associated with PDR. Based on the analysis of DEGs compared with the control samples, the significant genes associated with active FVMs and inactive FVMs were identified (Fig. 1). There were a total of 2,480 significantly dysregulated genes, including 690 upregulated and 1,790 downregulated genes, identified in the active FVMs. Furthermore, for the inactive FVMs a total of 2,369 genes were identified as significantly dysregulated, including 503 upregulated and 1,866 downregulated genes (Fig. 1A-C). According to the comparison of significant genes in each group, a total of 1,838 overlapping genes were identified as significant in active and inactive FVMs, comprising 642 specific genes for active FVMs and 531 specific genes for inactive FVMs. Among the overlapping genes, there were a total of 1,505 commonly downregulated genes and 332 commonly upregulated genes (Fig. 1D). Only one gene, flavin-containing monooxygenase isoform 2 (FMO2), was significantly downregulated $(\log \mathrm{FC}=-1.44, \mathrm{P}=0.00743)$ in the active FVMs but significantly upregulated $(\log F C=1.28$, $\mathrm{P}=0.000986$ ) in the inactive FVMs (Fig. 1E). FMO2 has been reported to exhibit catalytic functions in a diverse range of species $(19,20)$. In previous studies, the activation of FMO2 has been reported as necessary for dietary restriction-mediated life-span extension, and has also been reported as a biomarker for a starvation-associated longevity response in Caenorhabditis elegans $(21,22)$.

Functional enrichment associated with PDR. In order to annotate the functions of significant gene sets associated with PDR, KEGG pathway enrichment analysis was performed in each group of DEGs. In total, there were 24 significantly associated pathways in the active FVMs and 23 significantly associated pathways in the inactive FVMs, including 10 overlapping pathways (Fig. 2A). For the upregulated genes in the active FVMs, the top 10 pathways were extracellular matrix (ECM)-receptor interaction, focal adhesion, protein digestion and absorption, amoebiasis, leishmaniasis, small cell lung cancer, rheumatoid arthritis, cytokine-cytokine receptor interaction, pathways in cancer, and leukocyte transendothelial migration; for downregulated genes in the active FVMs, the top 10 pathways were metabolic pathways, tyrosine metabolism, arginine and proline metabolism, phototransduction, phenylalanine metabolism, $\beta$-alanine metabolism, proximal tubule bicarbonate reclamation, calcium signaling pathway, tryptophan metabolism, and histidine metabolism (Fig. 2B). By contrast, the top 10 pathways for upregulated genes in the inactive FVMs were ECM-receptor interaction, rheumatoid arthritis, cell adhesion molecules (CAMs), Staphylococcus aureus infection, graft-vs.-host disease, focal adhesion, asthma, intestinal immune network for IgA production, amoebiasis, and type 

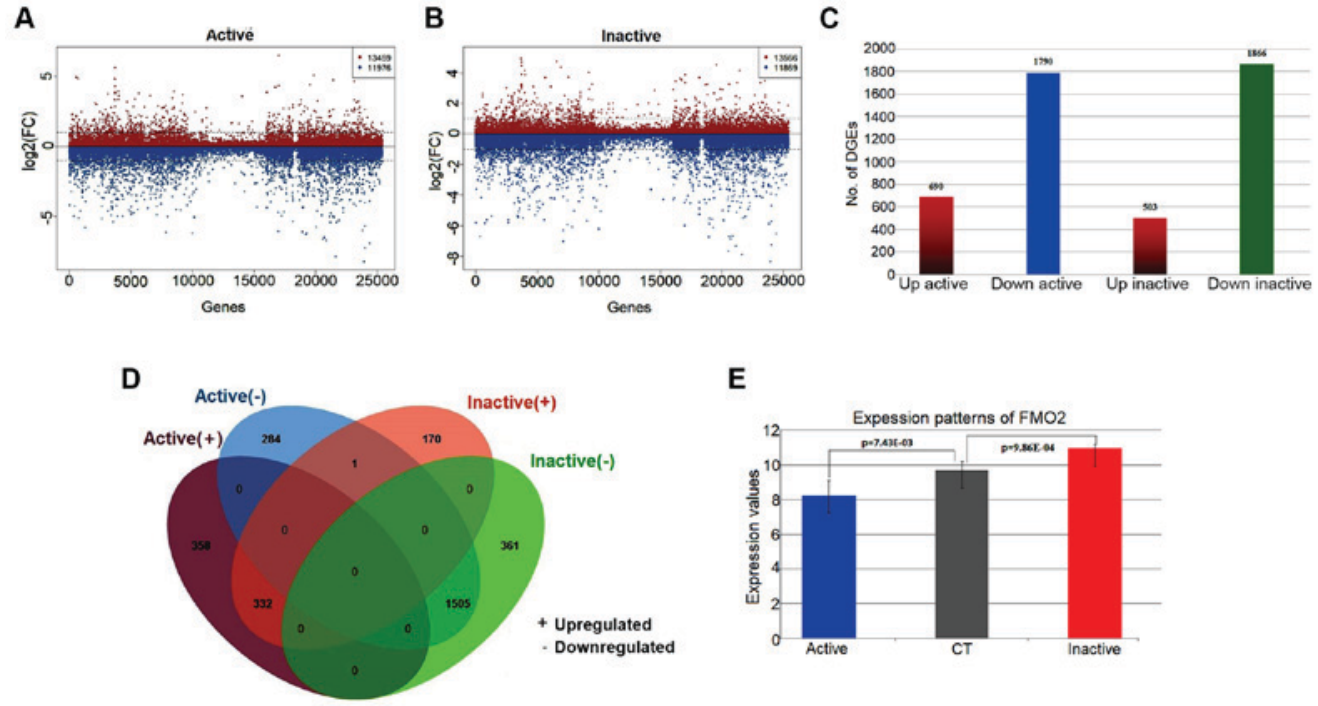

Figure 1. Overview of DEGs associated with PDR. (A) Scatter diagram illustrating $\log 2$ (FC) values of each gene between the active and control samples, including 13,459 upregulated genes and 11,976 downregulated genes. (B) Scatter diagram illustrating $\log 2(\mathrm{FC})$ values of each gene between the inactive and control samples, including 13,566 upregulated genes and 11,869 downregulated genes. (C) Histogram of the numbers of DEGs in the active and inactive samples. There were 690 upregulated genes and 1,790 downregulated genes identified as significant in the active samples. There were 503 upregulated genes and 1,866 downregulated genes significantly identified in the inactive samples. (D) Venn diagram illustrating the overlapping genes of DEGs in the active and inactive samples. (E) Bar chart of the expression patterns of FMO2 in the active and inactive samples. FMO2 was significantly downregulated (logFC=-1.44, $\mathrm{P}=0.00743)$ in the active $\mathrm{FVMs}$ but significantly upregulated $(\log \mathrm{FC}=1.28, \mathrm{P}=0.000986)$ in the inactive FVMs. PDR, proliferative diabetic retinopathy; FC, fold change; FVMs, fibrovascular membranes; FMO2, flavin-containing monooxygenase isoform 2; CT, control.

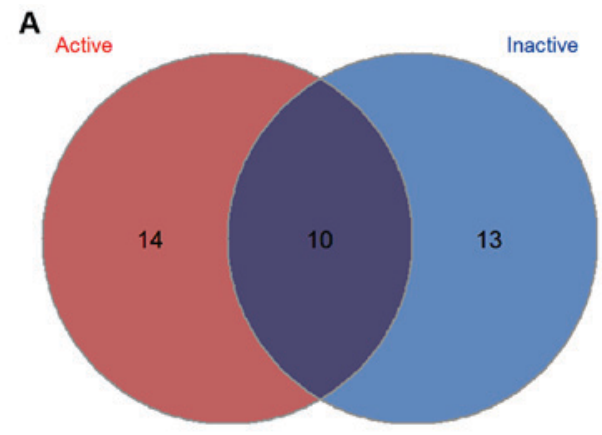

B

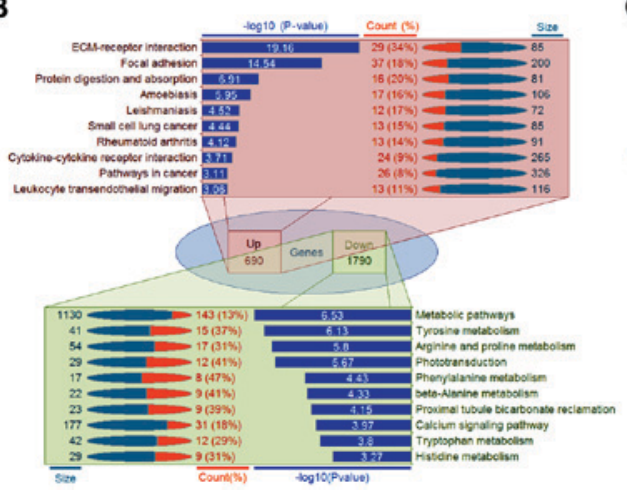

C

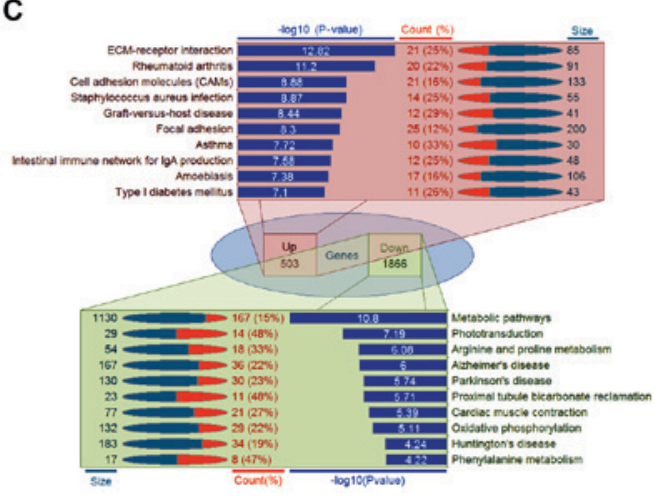

Figure 2. Significantly enriched pathways associated with PDR. (A) Venn diagram illustrating the overlapping pathways in the active and inactive samples. (B) Detailed information of the top 10 pathways in the active samples. For 690 upregulated genes in the active FVMs, the top 10 pathways were ECM-receptor interaction, focal adhesion, protein digestion and absorption, amoebiasis, leishmaniasis, small cell lung cancer, rheumatoid arthritis, cytokine-cytokine receptor interaction, pathways in cancer, and leukocyte transendothelial migration; for 1,790 downregulated genes in the active FVMs, the top 10 pathways were metabolic pathways, tyrosine metabolism, arginine and proline metabolism, phototransduction, phenylalanine metabolism, $\beta$-alanine metabolism, proximal tubule bicarbonate reclamation, calcium signaling pathway, tryptophan metabolism, and histidine metabolism. (C) Detailed information of the top 10 pathways in the inactive samples. For 503 upregulated genes in the active FVMs, the top 10 pathways were ECM-receptor interaction, focal adhesion, protein digestion and absorption, amoebiasis, leishmaniasis, small cell lung cancer, rheumatoid arthritis, cytokine-cytokine receptor interaction, pathways in cancer, and leukocyte transendothelial migration; for 1,866 downregulated genes in the active FVMs, the top 10 pathways were metabolic pathways, tyrosine metabolism, arginine and proline metabolism, phototransduction, phenylalanine metabolism, $\beta$-alanine metabolism, proximal tubule bicarbonate reclamation, calcium signaling pathway, tryptophan metabolism, and histidine metabolism. PDR, proliferative diabetic retinopathy; FVMs, fibrovascular membranes; Up, upregulated; Down, downregulated; ECM, extracellular matrix. 
Table I. Top 10 upregulated pathways in active fibrovascular membranes.

\begin{tabular}{|c|c|c|c|}
\hline Term & P-value & $\mathrm{n}$ & Gene symbols \\
\hline ECM-receptor interaction & $6.88 \mathrm{E}-20$ & 29 & $\begin{array}{l}\text { CD36, COL1A1, COL1A2, COL3A1, COL4A1, COL4A2, } \\
\text { COL5A1, COL5A2, COL6A1, COL6A2, COL6A3, FN1, } \\
\text { HMMR, HSPG2, TNC, ITGA1, ITGA2, ITGA4, ITGA5, } \\
\text { ITGB1, LAMA4, LAMB1, LAMC1, SPP1, THBS1, } \\
\text { THBS2, ITGA11, COL5A3, AGRN }\end{array}$ \\
\hline Focal adhesion & $2.86 \mathrm{E}-15$ & 37 & $\begin{array}{l}\text { ACTN1, BIRC2, CCND1, CAV1, COL1A1, COL1A2, } \\
\text { COL3A1, COL4A1, COL4A2, COL5A1, COL5A2, } \\
\text { COL6A1, COL6A2, COL6A3, DOCK1, FN1, TNC, ITGA1 } \\
\text { ITGA2, ITGA4, ITGA5, ITGB1, LAMA4, LAMB1, } \\
\text { LAMC1, PDGFB, PDGFRB, PGF, SPP1, THBS1, THBS2, } \\
\text { VEGFC, ZYX, ITGA11, COL5A3, PARVG, MYLK2, }\end{array}$ \\
\hline Protein digestion and absorption & $1.23 \mathrm{E}-07$ & 16 & $\begin{array}{l}\text { COL1A1, COL1A2, COL3A1, COL4A1, COL4A2, } \\
\text { COL5A1, COL5A2, COL6A1, COL6A2, COL6A3, } \\
\text { COL12A1, COL15A1, SLC1A5, COL5A3, COL18A1, } \\
\text { SLC36A1 }\end{array}$ \\
\hline Amoebiasis & $1.13 \mathrm{E}-06$ & 17 & $\begin{array}{l}\text { ACTN1, COL1A1, COL1A2, COL3A1, COL4A1, } \\
\text { COL4A2, COL5A1, COL5A2, FN1, IL1B, LAMA4, } \\
\text { LAMB1, LAMC1, PLCB2 TGFB3 TNF, COL5A3 }\end{array}$ \\
\hline Leishmaniasis & $3.00 \mathrm{E}-05$ & 12 & $\begin{array}{l}\text { CYBA, FCGR1 A, HLA-DMB, HLA-DOA, HLA-DQA1, } \\
\text { IL1B, ITGA4, ITGB1, STAT1, TGFB3, TNF, MARCKSL1 }\end{array}$ \\
\hline Small cell lung cancer & $3.61 \mathrm{E}-05$ & 13 & $\begin{array}{l}\text { BIRC2, CCND1, CDK6, CDKN2B, COL4A1, COL4A2, } \\
\text { E2F2, FN1, ITGA2, ITGB1, LAMA4, LAMB1, LAMC1 }\end{array}$ \\
\hline Rheumatoid arthritis & $7.51 \mathrm{E}-05$ & 13 & $\begin{array}{l}\text { CD28, HLA-DMB, HLA-DOA, HLA-DQA1, IL1B, PGF, } \\
\text { CCL2, CCL3, CCL3L1, TGFB3, TNF, TNFSF13B, CCL3L3 }\end{array}$ \\
\hline $\begin{array}{l}\text { Cytokine-cytokine receptor } \\
\text { interaction }\end{array}$ & $1.95 \mathrm{E}-04$ & 24 & $\begin{array}{l}\text { BMPR2, IL1B, IL2RB, INHBB, CXCL10, CXCL9, } \\
\text { TNFRSF11B, PDGFB, PDGFRB, CCL2, CCL3 CCL3L1, } \\
\text { CCL8, TGFB3, TNF, TNFSF4, TNFRSF4, VEGFC, } \\
\text { TNFRSF10D, TNFSF13B, TNFRSF21, TNFRSF12A, } \\
\text { CCL4L1, CCL3L3 }\end{array}$ \\
\hline Pathways in cancer & $7.78 \mathrm{E}-04$ & 26 & $\begin{array}{l}\text { BIRC2, BIRC5, CCND1, RUNX1, CDK6, CDKN2B, } \\
\text { COL4A1, COL4A2, E2F2, ETS1, FN1, ITGA2, ITGB1, } \\
\text { LAMA4, LAMB1, LAMC1, MMP9, PDGFB, PDGFRB, } \\
\text { PGF, RALA, STAT1, TGFB3, HSP90B1, VEGFC, LEF1 }\end{array}$ \\
\hline $\begin{array}{l}\text { Leukocyte transendothelial } \\
\text { migration }\end{array}$ & $8.65 \mathrm{E}-04$ & 13 & $\begin{array}{l}\text { ACTN1, CDH5, CYBA, CYBB, ITGA4, ITGB1, MMP9, } \\
\text { MSN, PECAM1, THY1, CLDN5, CLDN1, ESAM }\end{array}$ \\
\hline
\end{tabular}

I diabetes mellitus. The top 10 pathways for downregulated genes in the inactive FVMs were metabolic pathways, phototransduction, arginine and proline metabolism, Alzheimer's disease, Parkinson's disease, proximal tubule bicarbonate reclamation, cardiac muscle contraction, oxidative phosphorylation, Huntington's disease, and phenylalanine metabolism (Fig. 2C). The details of associated genes in these top 10 pathways in active and inactive FVMs are presented in Tables I and II.

TFs regulating target genes associated with PDR. By integrating the target genes identified in the present study with the gene regulation data from TRANSFAC, several associated TFs were identified for the active and inactive FVMs, respectively. As a result, a total of 118 TFs were identified for the active FVMs, including 26 upregulated
TFs and 92 downregulated TFs. For the inactive FVMs, a 103 total TFs were identified, including 20 upregulated and 83 downregulated TFs. According to the aforementioned data comparison, there were 78 overlapping TFs, including eight commonly upregulated and 70 commonly downregulated TFs (Fig. 3A). Among these, the transcription factor lymphoid enhancing binding factor-1 (LEF1) was identified as an important regulator. LEF1 is reported to be a central regulator of effecting the differentiation and cell number of invariant natural killer T cells (23). It has been revealed that the Wnt/ $\beta$-catenin effector LEF1 may regulate tyrosinase gene transcription during melanocyte development and differentiation (24). In the present study, LEF1 was upregulated in active and inactive FVMs, with the ability to activate nine target genes, including claudin 2 , catenin $\beta 1 \delta$-like 1, microphthalmia-associated transcription factor, 
Table II. Top 10 upregulated pathways in inactive fibrovascular membranes.

\begin{tabular}{|c|c|c|c|}
\hline Term & P-value & $\mathrm{n}$ & Gene symbols \\
\hline ECM-receptor interaction & $1.52 \mathrm{E}-13$ & 21 & $\begin{array}{l}\text { COL1A1, COL1A2, COL3A1, COL4A1, COL4A2, } \\
\text { COL5A1, COL5A2, COL6A1, COL6A3, COL11A1, } \\
\text { COMP, TNC, ITGA1, ITGA2, ITGA5, LAMA4, LAMB1, } \\
\text { LAMC1, THBS1, THBS2, ITGA11 }\end{array}$ \\
\hline Rheumatoid arthritis & $6.31 \mathrm{E}-12$ & 20 & $\begin{array}{l}\text { CD86, HLA-DMA, HLA-DMB, HLA-DOA, HLA-DPB1, } \\
\text { HLA-DQA1, HLA-DQB1, HLA-DRA, HLA-DRB4, IL1B, } \\
\text { IL6, ITGAL, PGF, CCL2, CCL3, CCL3L1, CCL5, TNF, } \\
\text { TNFSF13B, CCL3L3 }\end{array}$ \\
\hline Cell adhesion molecules (CAMs) & $1.31 \mathrm{E}-09$ & 21 & $\begin{array}{l}\text { CD2, CD6, CD8A, CD86, CD58, CDH2, HLA-DMA, } \\
\text { HLA-DMB, HLA-DOA, HLA-DPB1, HLA-DQA1, } \\
\text { HLA-DQB1, HLA-DRA, HLA-DRB4, ITGAL, NRCAM, } \\
\text { CLDN11, PECAM1, CLDN5, CLDN1, ICOS }\end{array}$ \\
\hline Staphylococcus aureus infection & $1.34 \mathrm{E}-09$ & 14 & $\begin{array}{l}\text { C3AR1, C5AR1, FCGR1A, CFH, HLA-DMA, HLA-DMB, } \\
\text { HLA-DOA, HLA-DPB1, HLA-DQA1, HLA-DQB1, } \\
\text { HLA-DRA, HLA-DRB4, CFI, ITGAL }\end{array}$ \\
\hline Graft-vs.-host disease & 3.67E-09 & 12 & $\begin{array}{l}\text { CD86, HLA-DMA, HLA-DMB, HLA-DOA, HLA-DPB1, } \\
\text { HLA-DQA1, HLA-DQB1, HLA-DRA, HLA-DRB4, IL1B, } \\
\text { IL6, TNF }\end{array}$ \\
\hline Focal adhesion & $4.98 \mathrm{E}-09$ & 25 & $\begin{array}{l}\text { ACTN1, COL1A1, COL1A2, COL3A1, COL4A1, } \\
\text { COL4A2, COL5A1, COL5A2, COL6A1, COL6A3, } \\
\text { COL11A1 COMP, TNC, ITGA1, ITGA2, ITGA5 LAMA4, } \\
\text { LAMB1, LAMC1, PGF, RAC2, THBS1, THBS2, ITGA11, } \\
\text { PARVG }\end{array}$ \\
\hline Asthma & $1.89 \mathrm{E}-08$ & 10 & $\begin{array}{l}\text { FCER1G, HLA-DMA, HLA-DMB, HLA-DOA, } \\
\text { HLA-DPB1, HLA-DQA1, HLA-DQB1, HLA-DRA, } \\
\text { HLA-DRB4, TNF }\end{array}$ \\
\hline Intestinal immune network for & $2.65 \mathrm{E}-08$ & 12 & CD86, HLA-DMA, HLA-DMB, HLA-DOA, HLA-DPB 1, \\
\hline IgA production & & & $\begin{array}{l}\text { HLA-DQA1, HLA-DQB1, HLA-DRA, HLA-DRB4, IL6, } \\
\text { TNFSF13B, ICOS }\end{array}$ \\
\hline Amoebiasis & $4.21 \mathrm{E}-08$ & 17 & $\begin{array}{l}\text { ACTN1, CD14 COL1A1, COL1A2, COL3A1, COL4A1, } \\
\text { COL4A2, COL5A1, COL5A2, COL11A1, IL1B, IL6, } \\
\text { LAMA4, LAMB1, LAMC1, PLCB2, TNF }\end{array}$ \\
\hline Type I diabetes mellitus & $7.95 \mathrm{E}-08$ & 11 & $\begin{array}{l}\text { CD86, HLA-DMA, HLA-DMB, HLA-DOA, HLA-DPB1, } \\
\text { HLA-DQA1, HLA-DQB1, HLA-DRA, HLA-DRB4, IL1B, } \\
\text { TNF }\end{array}$ \\
\hline
\end{tabular}

matrix metalloproteinase 7, MYC binding protein, neuronal cell adhesion molecule, OCA2 and secreted phosphoprotein 1 (SPP1). However, the transcription of six genes, namely ALX4, CD1D, desmoglein 4, interleukin (IL)13, IL4 and IL5, were repressed by LEF1. The majority of LEF1 target genes had similar expression levels between active and inactive FVMs. However, the expression of SPP1 was significantly higher in the active samples (Fig. 3B), but was not significantly altered in the inactive samples (Fig. 3C), when compared with the controls. The co-expression of SPP1 and LEF1 protein was previously investigated by immunohistochemistry using a tFVM microarray, which demonstrated that LEF1 may serve as a prognostic biomarker for primary colorectal carcinoma and liver metastases (25). In the present study, it was demonstrated that the Wnt/ $\beta$-catenin effector LEF1 regulating SPP1 may be important in PDR.
In a previous study based on microarray analysis using Welch's t-test coupled with a multidimensional false-discovery control by Ishikawa et al (3), only 91 genes were identified to be significantly upregulated in active FVMs, the majority of which were clustered in the functional category of angiogenesis. A total of 89 genes were shown to be significantly upregulated in inactive FVMs, the majority of which were clustered in the functional category of metabolism. Furthermore, the ingenuity pathway analysis revealed that ECM-related molecules, including periostin, tenascin $\mathrm{C}$, hexabrachion, transforming growth factor $\beta$ families, and angiogenic factors, are essential in promoting the development of FVMs associated with PDR. These genetic factors were also identified in the list of upregulated genes in active and inactive FVMs in the present study. A number of previously unreported genes were reidentified, therefore, these results appeared to be more 
B

A
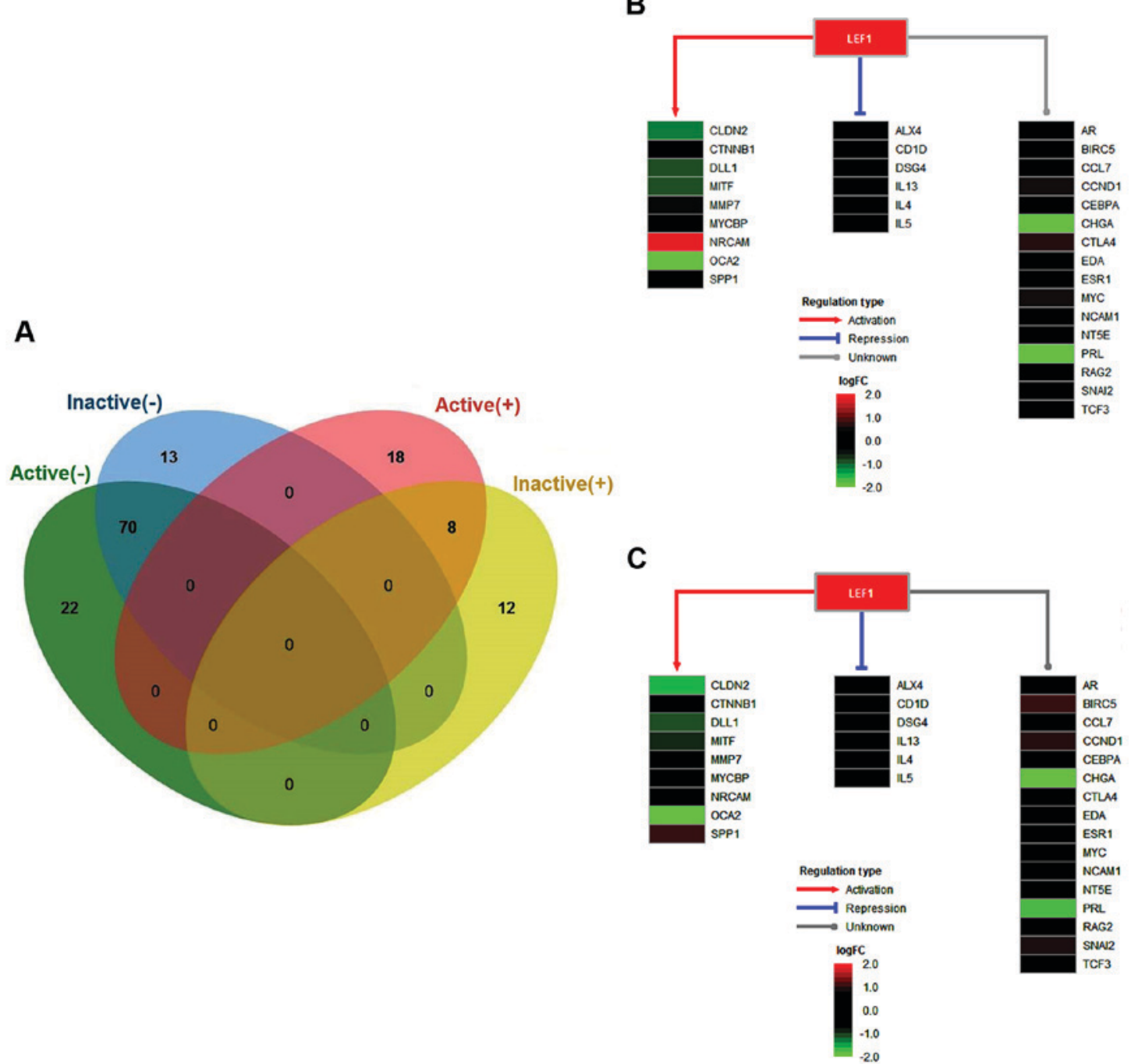

Figure 3. Transcription factors associated with proliferative diabetic retinopathy. (A) Venn diagram illustrating the overlapping key TFs in the active and inactive samples. (B) Regulatory model of LEF1 in the active samples, including the regulation types of activation, repression, and unknown. (C) Regulatory model of LEF1 in the inactive samples, including the regulation types of activation, repression, and unknown. TFs, transcription factors; LEF1, lymphoid enhancing binding factor-1.

systematic and comprehensive, when compared with those of previous studies. However, due to the lack of relevant molecular biological experimental data in the present study, the identification of genetic site information requires further confirmation in future investigations.

\section{Acknowledgements}

Not applicable.

\section{Funding}

The present study was supported by the Key University Science Research Project of Anhui Province (grant no. KJ2017A021), the Natural Science Foundation Project of Anhui Province (grant no. 1508085QC63), the Scientific Research Foundation and Academic \& Technology Leaders Introduction Project, and 211 Project of Anhui University (grant no. 10117700023), the Student Research Training Program of Anhui University (grant nos. J10118520218 and J10118520307) and the
Education Revitalization Project of Anhui Province: Stem Cell and Translational Medicine (grant no. Y05201374).

\section{Availability of data and materials}

The datasets used and/or analyzed during the current study are available from the corresponding author on reasonable request.

\section{Authors' contributions}

MTG and KH designed the study. WXL, QZ, ZHH, SLZ and WWL performed breast cancer data collection. HZ, JW and MTG performed data analysis. WXL, QZ, KH and MTG wrote the manuscript. The final version of the manuscript has been read and approved by all authors, and each author considers the manuscript to represent honest work.

\section{Ethics approval and consent to participate}

Not applicable. 


\section{Consent for publication}

Not applicable.

\section{Competing interests}

The authors declare that they have no competing interests.

\section{References}

1. Graham PS, Kaidonis G, Abhary S, Gillies MC, Daniell M, Essex RW, Chang JH, Lake SR, Pal B, Jenkins AJ, et al: Genome-wide association studies for diabetic macular edema and proliferative diabetic retinopathy. BMC Med Genet 19: 71, 2018.

2. Huang YC, Liao WL, Lin JM, Chen CC, Liu SP, Chen SY, Lin YN, Lei YJ, Liu HT, Chen YJ and Tsai FJ: High levels of circulating endothelial progenitor cells in patients with diabetic retinopathy are positively associated with ARHGAP22 expression. Oncotarget 9: 17858-17866, 2018.

3. Ishikawa K, Yoshida S, Kobayashi Y, Zhou Y, Nakama T, Nakao S, Sassa Y, Oshima Y, Niiro H, Akashi K, et al: Microarray analysis of gene expression in fibrovascular membranes excised from patients with proliferative diabetic retinopathy. Invest Ophthalmol Vis Sci 56: 932-946, 2015.

4. Nathan DM: Long-term complications of diabetes mellitus. N Engl J Med 328: 1676-1685, 1993.

5. Uhlmann K, Kovacs P, Boettcher Y, Hammes HP and Paschke R Genetics of diabetic retinopathy. Exp Clin Endocrinol Diabetes 114: 275-294, 2006.

6. Khalfaoui T, Lizard G, Beltaief O, Colin D, Ben Hamida J, Errais K, Ammous I, Zbiba W, Tounsi L, Zhioua R, et al: Immunohistochemical analysis of cellular adhesion molecules (ICAM-1, VCAM-1) and VEGF in fibrovascular membranes of patients with proliferative diabetic retinopathy: Preliminary study. Pathol Biol (Paris) 57: 513-517, 2009.

7. Beranek M, Kolar P, Tschoplova S, Kankova K and Vasku A: Genetic variation and plasma level of the basic fibroblast growth factor in proliferative diabetic retinopathy. Diabetes Res Clin Pract 79: 362-367, 2008.

8. Uthra S, Raman R, Mukesh BN, Rajkumar SA, Kumari RP, Agarwal S, Paul PG, Lakshmipathy P, Gnanamoorthy P, Sharma T, et al: Diabetic retinopathy and IGF-1 gene polymorphic cytosine-adenine repeats in a Southern Indian cohort. Ophthalmic Res 39: 294-299, 2007.

9. Cilenšek I, Mankoč S, Globočnik Petrovič M and Petrovič D: The 4a/4a genotype of the VNTR polymorphism for endothelial nitric oxide synthase (eNOS) gene predicts risk for proliferative diabetic retinopathy in Slovenian patients (Caucasians) with type 2 diabetes mellitus. Mol Biol Rep 39: 7061-7067, 2012.

10. Crispim D, Fagundes NJ, dos Santos KG, Rheinheimer J, Bouças AP, de Souza BM, Macedo GS, Leiria LB, Gross JL and Canani LH: Polymorphisms of the UCP2 gene are associated with proliferative diabetic retinopathy in patients with diabetes mellitus. Clin Endocrinol (Oxf) 72: 612-619, 2010.

11. Santos KG, Crispim D, Canani LH, Ferrugem PT, Gross JL and Roisenberg I: Relationship of endothelial nitric oxide synthase (eNOS) gene polymorphisms with diabetic retinopathy in Caucasians with type 2 diabetes. Ophthalmic Genet 33: 23-27, 2012.
12. Choi D, Appukuttan B, Binek SJ, Planck SR, Stout JT, Rosenbaum JT and Smith JR: Prediction of Cis-regulatory elements controlling genes differentially expressed by retinal and choroidal vascular endothelial cells. J Ocul Biol Dis Infor 1: 37-45, 2008.

13. Gu Y, Ke G, Wang L, Zhou E, Zhu K and Wei Y: Altered expression profile of circular RNAs in the serum of patients with diabetic retinopathy revealed by microarray. Ophthalmic Res 58: 176-184, 2017.

14. Edgar R, Domrachev M and Lash AE: Gene expression omnibus: NCBI gene expression and hybridization array data repository. Nucleic Acids Res 30: 207-210, 2002.

15. Carvalho BS and Irizarry RA: A framework for oligonucleotide microarray preprocessing. Bioinformatics 26 : 2363-2367, 2010.

16. Huang da W, Sherman BT and Lempicki RA: Systematic and integrative analysis of large gene lists using DAVID bioinformatics resources. Nat Protoc 4: 44-57, 2009.

17. Matys V, Kel-Margoulis OV, Fricke E, Liebich I, Land S, Barre-Dirrie A, Reuter I, Chekmenev D, Krull M, Hornischer K, et al: TRANSFAC and its module TRANSCompel: Transcriptional gene regulation in eukaryotes. Nucleic Acids Res 34 (Database Issue): D108-D110, 2006.

18. Wingender E, Dietze P, Karas H and Knüppel R: TRANSFAC: A database on transcription factors and their DNA binding sites. Nucleic Acids Res 24: 238-241, 1996.

19. Karoly ED and Rose RL: Sequencing, expression, and characterization of cDNA expressed flavin-containing monooxygenase 2 from mouse. J Biochem Mol Toxicol 15: 300-308, 2001.

20. Scharf ME, Scharf DW, Bennett GW and Pittendrigh BR: Catalytic activity and expression of two flavin-containing monooxygenases from Drosophila melanogaster. Arch Insect Biochem Physiol 57: 28-39, 2004.

21. Bennett CF, Kwon JJ, Chen C, Russell J, Acosta K, Burnaevskiy N, Crane MM, Bitto A, Vander Wende H, Simko M, et al: Transaldolase inhibition impairs mitochondrial respiration and induces a starvation-like longevity response in Caenorhabditis elegans. PLoS Genet 13: e1006695, 2017.

22. Leiser SF, Miller H, Rossner R, Fletcher M, Leonard A, Primitivo M, Rintala N, Ramos FJ, Miller DL and Kaeberlein M: Cell nonautonomous activation of flavin-containing monooxygenase promotes longevity and health span. Science 350 : 1375-1378, 2015.

23. Carr T, Krishnamoorthy V, Yu S, Xue HH, Kee BL and Verykokakis M: The transcription factor lymphoid enhancer factor 1 controls invariant natural killer $\mathrm{T}$ cell expansion and Th2-type effector differentiation. J Exp Med 212: 793-807, 2015.

24. Wang X, Liu Y, Chen H, Mei L, He C, Jiang L, Niu Z, Sun J, Luo $\mathrm{H}, \mathrm{Li} \mathrm{J}$ and Feng $\mathrm{Y}$ : LEF-1 regulates tyrosinase gene transcription in vitro. PLoS One 10: e0143142, 2015.

25. Lin AY, Chua MS, Choi YL, Yeh W, Kim YH, Azzi R, Adams GA, Sainani K, van de Rijn M, So SK and Pollack JR: Comparative profiling of primary colorectal carcinomas and liver metastases identifies LEF1 as a prognostic biomarker. PLoS One 6: e16636, 2011. 